Article

\title{
Numerical Simulation of Electromagnetic Field in Round Bloom Continuous Casting with Final Electromagnetic Stirring
}

\author{
Bingzhi Ren ${ }^{1,2}{ }^{(\mathbb{D}}$, Dengfu Chen ${ }^{2, *}$, Wentang Xia ${ }^{1}$, Hongdan Wang ${ }^{1, *(1)}$ and Zhiwei Han ${ }^{3}$ \\ 1 School of Metallurgical and Materials Engineering, Chongqing University of Science \& Technology, \\ Chongqing 401331, China; renbingzhi@cqust.edu.cn (B.R.); wentangx@163.com (W.X.) \\ 2 College of Materials Science and Engineering, Chongqing University, Chongqing 400044, China \\ 3 Department of Continuous Casting, CISDI Engineering Co. Ltd., Chongqing 400013, China; \\ zhiwei.han@cisdi.com.cn \\ * Correspondence: chendfu@cqu.edu.cn (D.C.); wanghongdan@cqust.edu.cn (H.W.); \\ Tel.: +86-023-6510-2467 (D.C.); +86-023-6502-3706 (H.W.)
}

Received: 25 September 2018; Accepted: 1 November 2018; Published: 5 November 2018

\begin{abstract}
A 3D mathematical model was developed to simulate the electromagnetic field in $\Phi 600 \mathrm{~mm}$ round bloom continuous casting with final electromagnetic stirring (F-EMS), and the model was verified using measured data for the magnetic flux density in the stirrer centre. The distribution of electromagnetic force and the influence of current intensity and frequency were investigated. The results show that the Joule heat generated by F-EMS is very small and its influence on secondary cooling heat transfer in the stirring zone can be ignored. With an increase in current frequency, the electromagnetic force density at $R / 2$ and $R / 3$ of the $\Phi 600 \mathrm{~mm}$ round bloom first increases and then decreases, reaching a maximum at $10 \mathrm{~Hz}$.
\end{abstract}

Keywords: numerical simulation; round bloom; continuous casting; final electromagnetic stirring; electromagnetic field

\section{Introduction}

Continuous casting is a process generally used in steel production to make the molten steel solidified into a semi-finished billet, bloom, or slab for subsequent rolling in the finishing mills. Electromagnetic stirring (EMS) technology is widely used in the continuous casting production of steel. This technology utilises electromagnetic induction to provide a non-contact electromagnetic force to enhance the molten steel flow, heat transfer, and mass transfer and to promote columnar to equiaxed transition, thereby rectifying internal defects such as central segregation and shrinkage cavities [1]. According to different installation positions, three types of EMS exist in continuous casting steel. One is situated at the caster mould, referred to as mould electromagnetic stirring (M-EMS); another is situated along the strand in the secondary cooling zone, called a strand electromagnetic stirring (S-EMS); the last one is situated near the solidification end of the strand, known as final electromagnetic stirring (F-EMS) [2]. M-EMS is used in almost all billet/bloom casters. However, for high-carbon steel or large-section strands, the use of M-EMS cannot completely improve the internal quality of the strand, and F-EMS is generally required [3], which can more effectively improve the central porosity and V-shaped segregation of the strand [4,5]. Therefore, detailed analysis of the electromagnetic field, fluid flow, and solidification behaviour of the strand is very important for evaluating the impact of F-EMS on various metallurgical operations in the continuous casting process, and the electromagnetic field is the primary problem. 
In 1986, Spitzer et al. deduced the analytical formula for the 2D electromagnetic force in an infinitely long stirring system [6]. Recently, Vynnycky [7] derived another form of analytical solution for the same issue with Ref. [6] when the tangential magnetic flux density was employed as the boundary condition. Trindade et al. [8], Yu et al. [9], and Liu et al. [10] studied the electromagnetic field of M-EMS, highlighting the obvious shielding effect of mould copper on the electromagnetic field, while that of F-EMS is different. Jiang et al. [11] and Sun et al. [3] focused mainly on fluid flow, solidification, and mass transfer of the continuous casting strand with F-EMS and rarely discussed the electromagnetic field. Therefore, this study aims to investigate the electromagnetic field in $\$ 600 \mathrm{~mm}$ round bloom continuous casting with F-EMS by numerical simulation, as well as examining the distribution features of the electromagnetic force and the influence of current intensity and frequency.

\section{Mathematical Model}

The continuous caster studied in this paper is produced by a domestic steel mill (Sunan Heavy Industry, Suzhou, China). Figure 1 is a structural diagram of the continuously cast round bloom with F-EMS. The origin of the coordinate is at the centre of the stirrer. The round bloom has a diameter of $600 \mathrm{~mm}$ and the stirrer core has a height of $300 \mathrm{~mm}$.

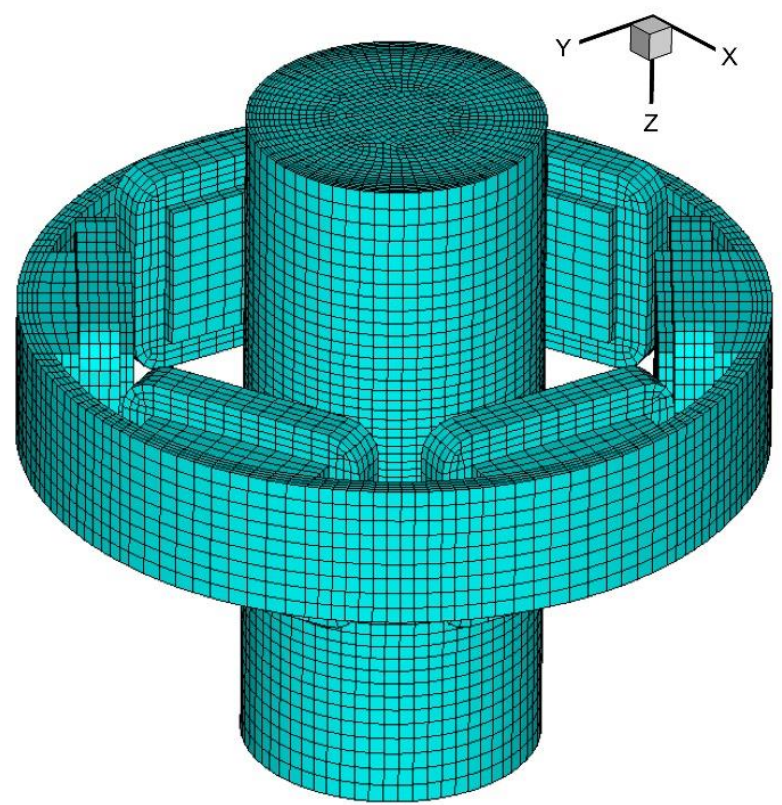

Figure 1. Schematic illustration of final electromagnetic stirring (F-EMS) for round bloom continuous casting. The air cylinder is not shown.

\subsection{Basic Assumption}

The fluid flow in a continuous casting round bloom with F-EMS can be described by Maxwell's equations and the Navier-Stokes equations, which are coupled by the electromagnetic force density and the flow velocity of the molten steel. In view of the complexity of the F-EMS process, several assumptions must be made in order to simplify the calculations.

1. In the F-EMS process, the magnetic Reynolds number (about 0.1) is far less than the shielding parameter (about 10) $[10,12]$ and the angular frequency of the current intensity is much larger than the angular velocity of the molten steel; thus, the influence of the molten steel flow on the electromagnetic field is negligible [13]. Therefore, this problem can be decomposed into an electromagnetic problem and a fluid flow problem to be solved separately. In the present paper, only the electromagnetic field was calculated without regard to the flow field and solidification. 
2. Since the current frequency of F-EMS is generally in the range of $4 \mathrm{~Hz}$ to $10 \mathrm{~Hz}$, which belongs to the magnetic quasi-static field, the displacement current is ignored [8].

3. Both the outer shell and the cooling water jacket of the stirrer are approximated as an air zone for the benefit of the calculations.

4. The curve of the strand is negligible.

\subsection{Control Equations}

At low current frequencies, the time-varying electromagnetic field is assumed to be a magnetic quasi-static field, and Maxwell's equations can be simplified as follows [8]:

$$
\begin{gathered}
\nabla \cdot \boldsymbol{B}=0 \\
\nabla \times \boldsymbol{B}=\mu_{\mathrm{r}} \mu_{0} \boldsymbol{J} \\
\nabla \times \boldsymbol{E}=-\frac{\partial \boldsymbol{B}}{\partial t} \\
\boldsymbol{J}=\sigma \boldsymbol{E}
\end{gathered}
$$

where $B$ is the magnetic flux density, $J$ is the induced current density in the strand, $\mu_{0}$ is the vacuum permeability, $\mu_{\mathrm{r}}$ is the relative permeability, $E$ is the electric field intensity, $\sigma$ is the electrical conductivity, and $t$ is the time.

The electromagnetic force density at each element is calculated by harmonic analysis using Equation (5) [8]:

$$
F_{\text {time }}=J \times B
$$

The expression for the Joule heat power density generated by the induced current is calculated from Equation (6) [14]:

$$
P_{\text {time }}=|\boldsymbol{J}|^{2} / \sigma
$$

The time in Equations (5) and (6) refers to the real and imaginary parts of the variable at $0^{\circ}$ and $90^{\circ}$, respectively.

Since F-EMS uses a sinusoidal current to generate a harmonic electromagnetic field, the magnetic flux density, electromagnetic force density and Joule heat power density change with time. However, at low current frequencies, both the shielding parameter and the interaction parameter are small, and it is feasible to use the time-averaged electromagnetic force density and Joule heat power density [15]. Hence, the time-averaged values of the two variables are calculated from Equations (7) and (8) in the post-processing to investigate the distribution characteristics [8]:

$$
\begin{aligned}
& \boldsymbol{F}_{\mathrm{em}}=\left(\boldsymbol{F}_{0}+\boldsymbol{F}_{90}\right) / 2 \\
& P_{\text {ave }}=\left(P_{0}+P_{90}\right) / 2
\end{aligned}
$$

Because the time-averaged value of the magnetic flux density is 0 , the time-averaged value of its modulus is transiently calculated by using Equation (9):

$$
B_{\text {mag }}=f \int_{t_{0}}^{t_{0}+1 / f}|\boldsymbol{B}| \mathrm{d} t
$$

where $f$ is the current frequency. 


\subsection{Boundary Conditions}

An air cylinder with a radius of $0.9 \mathrm{~m}$ and a height of $2.2 \mathrm{~m}$ was established around the whole stirring system to calculate the electromagnetic field. The tangential magnetic condition $(\partial \Phi / \partial n)=0$ was applied at the outer surface of the air cylinder, where $\Phi$ is the reduced scalar potential and $n$ is the normal unit vector to the surface $[8,10]$. The three-phase coil excitation was applied with source current intensity $(I)$ and frequency.

\subsection{Physical Properties}

Before the $\Phi 600 \mathrm{~mm}$ round bloom is completely solidified, its temperature is above $1173 \mathrm{~K}$ [16], which is greater than the Curie point of the steel (about $1023 \mathrm{~K}$ ) [17]. Therefore, the strand is a paramagnetic material. In the calculation, it is considered that both the strand and the iron core of the stirrer are isotropic materials and that their relative permeability is constant. The relevant physical properties are as follows: the vacuum permeability is $1.257 \times 10^{-6} \mathrm{H} \cdot \mathrm{m}^{-1}$, the relative permeability of the strand and air is 1.0, the relative permeability of the iron core is 1000 , and the electrical conductivity of the strand is $7.14 \times 10^{5} \mathrm{~S} \cdot \mathrm{m}^{-1}$.

\subsection{Solution}

When calculating the electromagnetic field, the length of the strand is $1.8 \mathrm{~m}$. ANSYS Emag software (ANSYS 14.0, ANSYS Inc., Canonsburg, PA, USA) was used to solve Maxwell's equations by the finite element method (FEM). We employed three different meshes to verify the independence of the grid, which included 220,000, 270,000, and 350,000 elements, respectively. The comparison between simulation results and experimental data indicated that the errors of the three meshes are $7.2 \%, 4.6 \%$, and $4.0 \%$, respectively. Hence, a mesh of 270,000 elements was selected to obtain the mesh-independent results. The distribution of the electromagnetic field in the strand was obtained and the time-averaged electromagnetic force density was extracted. Except for the transient analysis of $B_{\text {mag }}$, other variables were calculated using harmonic analysis.

\section{Results and Discussion}

In order to validate the mathematical model of the electromagnetic field, the magnetic flux density in the stirrer was measured along the axial direction without the strand and compared with the numerical simulation results, as shown in Figure 2. The measurement of the magnetic flux density was carried out in a $\Phi 600 \mathrm{~mm}$ round bloom continuous caster at a domestic steel mill by using a portable Gauss meter (Bell, FW5180). The calculated values agree well with the measured data, which supports the correctness of the mathematical model and the solution method for the electromagnetic field. Figure 2 also demonstrates that the magnetic flux density in the stirrer with the strand is significantly lower than that without the strand, and the maximum value decreases from $55.4 \mathrm{mT}$ calculated without the strand to $51.1 \mathrm{mT}$ with the strand. This is mainly due to the 'skin effect': the strand can shield the electromagnetic field, resulting in a decrease in magnetic flux density.

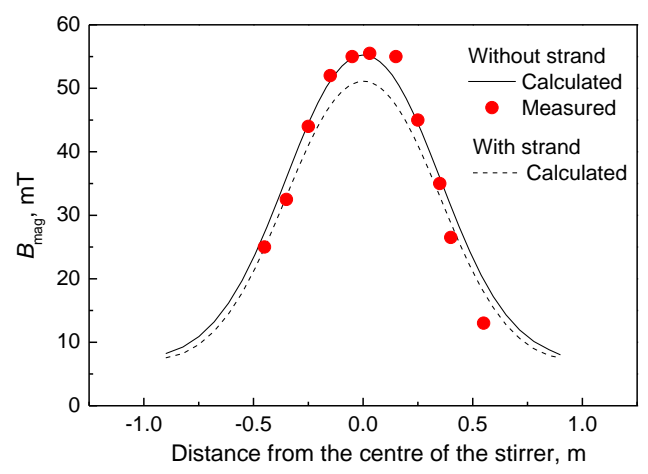

Figure 2. Profile of the magnetic flux density along the axial direction for $I=350 \mathrm{~A}$ and $f=4 \mathrm{~Hz}$. 
Figure 3 shows the distribution of the magnetic flux density in both the cross-section and the longitudinal section of the $\Phi 600 \mathrm{~mm}$ round bloom at $400 \mathrm{~A}$ and $4 \mathrm{~Hz}$. The magnetic flux density in the cross-section is roughly circularly symmetrical, and it is greatest at the edge and gradually attenuates towards the centre, as shown in Figure 3a. In the longitudinal section, the maximum value of the magnetic flux density appears in the middle of the stirrer and gradually weakens towards both ends, as shown in Figure 3b.
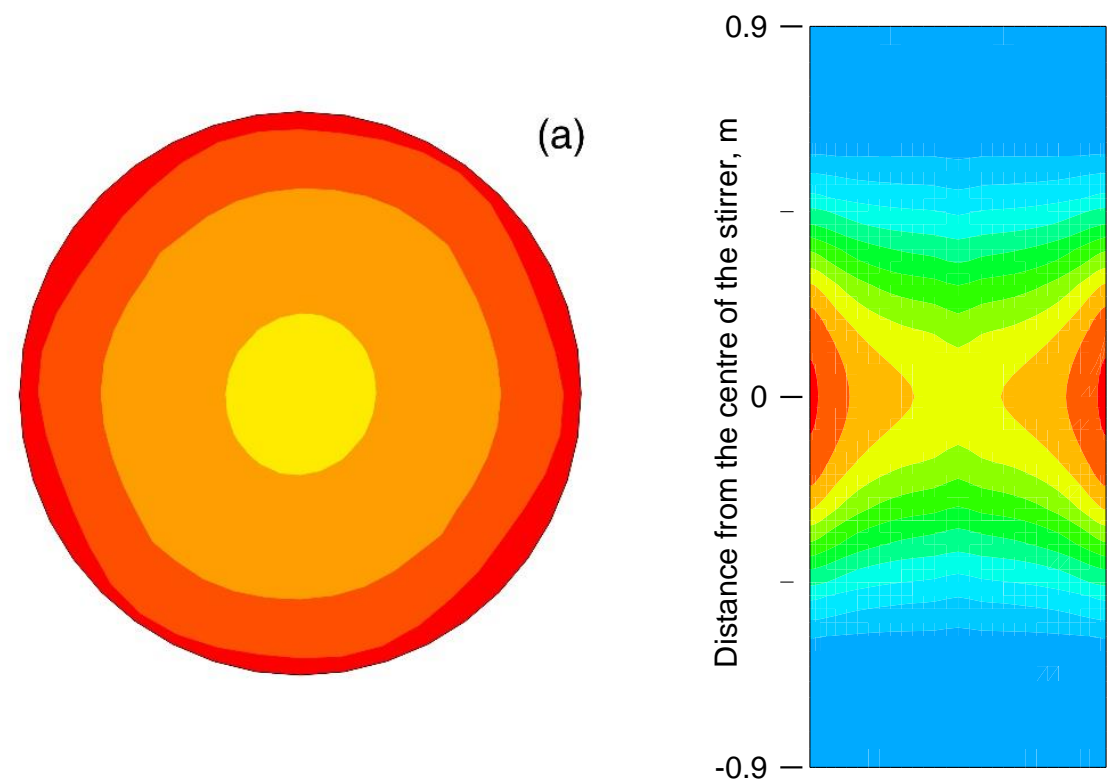

(b)

$B_{\text {mag }}, \mathrm{mT}$

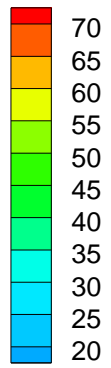

Figure 3. Contour plot of the magnetic flux density in the (a) cross-section and (b) longitudinal section for $I=400$ A and $f=4 \mathrm{~Hz}$.

Figure 4 displays the distribution of the time-averaged Joule heat power density in both the cross-section and the longitudinal section of the $\Phi 600 \mathrm{~mm}$ round bloom at $400 \mathrm{~A}$ and $4 \mathrm{~Hz}$. The characteristic of the Joule heat power density distribution is very similar to that of the magnetic flux density. It can also be seen from Figure $4 a$ that the distribution of Joule heat power density has a hexagonal symmetry at the edge of the strand. This is because the iron core of the stirrer has six protruding tooth parts (see Figure 1), and the Joule heat power density at the edge of the strand near to them is large. Through the integral calculation, it is found that the Joule heat generated by F-EMS in the strand is $5.3 \mathrm{~kW}$, and the quantity of the secondary cooling heat transfer $(P)$ in this region is about $201 \mathrm{~kW}$, which can be approximately calculated using Equation (10) [18]. Therefore, the Joule heat is small and negligible compared with the secondary cooling heat transfer in the stirring zone.

$$
P=\varepsilon \delta\left(T_{\mathrm{S}}^{4}-T_{0}^{4}\right) C H / 1000
$$

where $\varepsilon$ is the emissivity of the steel ( 0.85 in the present study), $\delta$ is the Stefan-Boltzmann constant $\left(5.67 \times 10^{-8} \mathrm{~W} \cdot \mathrm{m}^{-2} \cdot \mathrm{K}^{-4}\right), T_{\mathrm{s}}$ is the surface temperature of the round bloom (about $\left.1223 \mathrm{~K}\right), T_{0}$ is the environmental temperature (about $373 \mathrm{~K}$ ), $C$ is the circumference of the round bloom, and $H$ is the height of the stirring zone, which is $1 \mathrm{~m}$. 


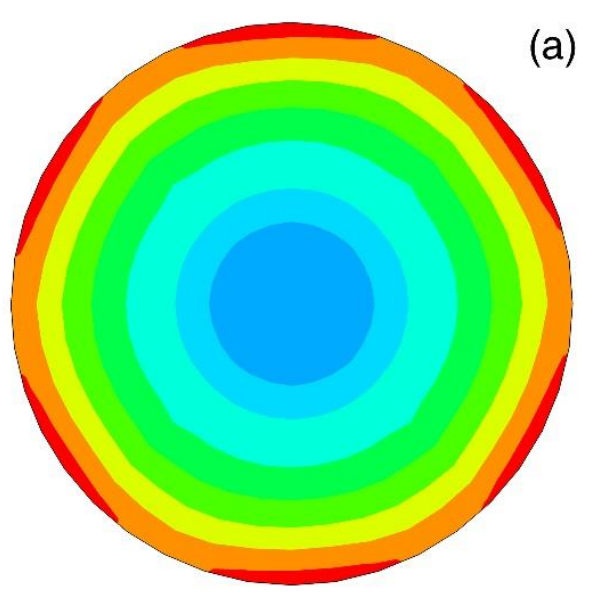

(a)

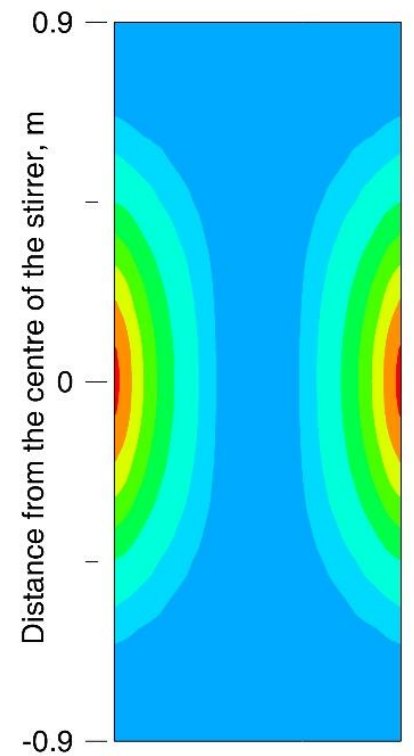

(b)

$P_{\text {ave }}, \mathrm{W} \cdot \mathrm{m}^{-3}$

60000

50000

40000

30000

20000

10000

5000

Figure 4. Contour plot of the time-averaged Joule heat power density in the (a) cross-section and

(b) longitudinal section for $I=400 \mathrm{~A}$ and $f=4 \mathrm{~Hz}$.

Figure 5a demonstrates the vector distribution of the time-averaged electromagnetic force density in the cross-section of the $\Phi 600 \mathrm{~mm}$ round bloom. The electromagnetic force density is generally distributed circumferentially, thereby causing the rotation of the molten steel. It is greatest at the edge and decreases significantly towards the centre. Figure $5 \mathrm{~b}$ shows the contour plot of the electromagnetic force density in the longitudinal section, which has a distribution similar to the magnetic flux density as well.
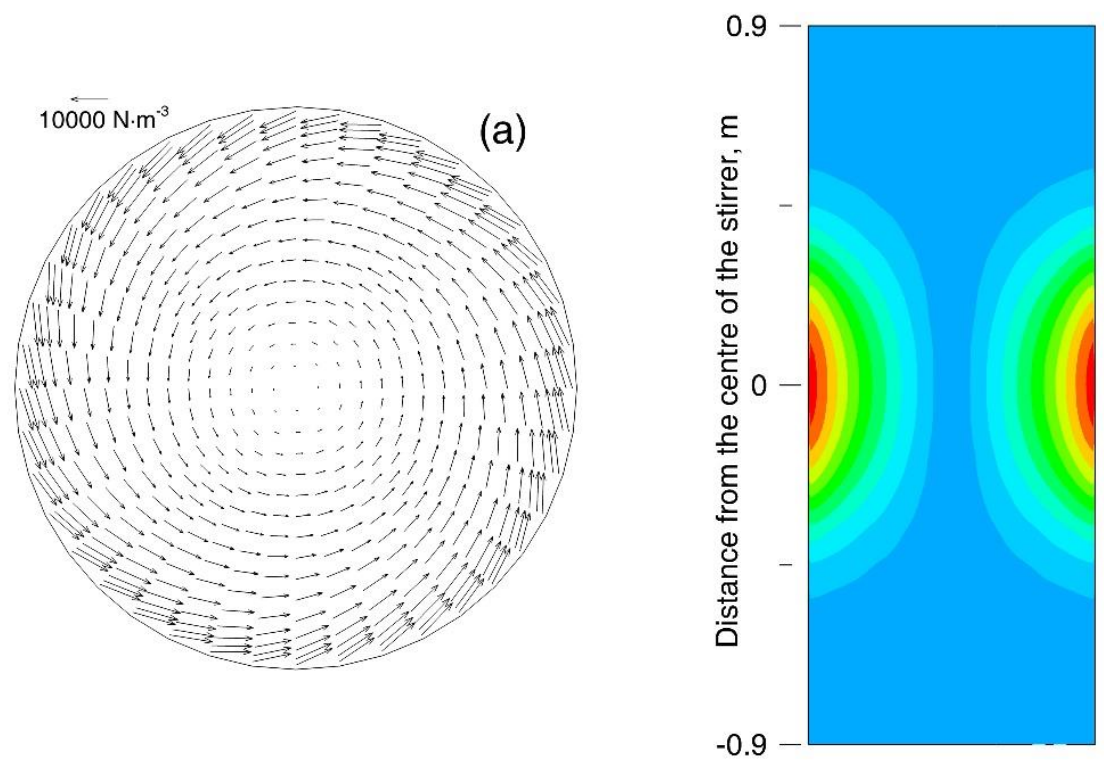

(b)

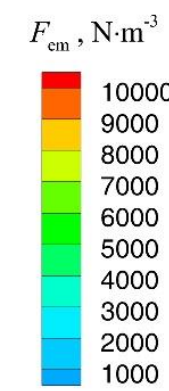

Figure 5. Distribution of the time-averaged electromagnetic force density in the (a) cross-section and (b) longitudinal section for $I=400 \mathrm{~A}$ and $f=4 \mathrm{~Hz}$.

Figure 6 compares the electromagnetic force density calculated by FEM with that obtained from the analytical formula of the infinite-length stirring system deduced by Spitzer et al. [6]. Among the three components of the electromagnetic force density, the tangential electromagnetic force density is the largest, which is also the driving force of molten steel rotation. The tangential electromagnetic force density calculated by FEM is greater than that calculated by the analytical formula, especially at 
the edge of the strand. However, in the effective area of F-EMS on the strand $(r<\mathrm{R} / 2)$, the difference between the two results is not large. In addition, the difference between the radial electromagnetic force densities calculated by FEM and by the analytical formula is also not large. Finally, the axial electromagnetic force density calculated by FEM is close to zero and negligible. In short, since the electromagnetic force generated by F-EMS usually acts in a region smaller than R/2 in a round bloom, it can be approximately calculated by the above analytical formula for the $\Phi 600 \mathrm{~mm}$ round bloom.

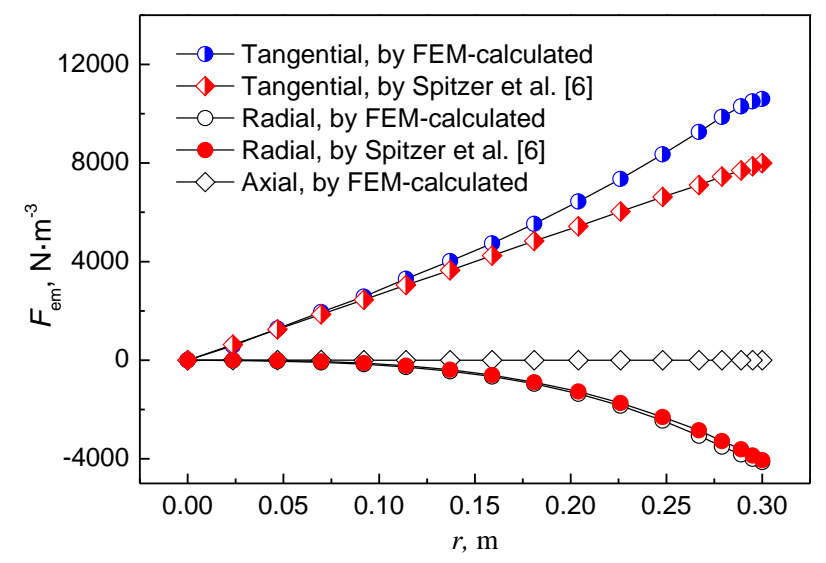

Figure 6. Comparison of FEM-calculated electromagnetic force density with that of the analytical solution by Spitzer et al. [6] in the middle plane of the stirrer for $I=400 \mathrm{~A}$ and $f=4 \mathrm{~Hz}$.

Figure 7 illustrates the variation in the electromagnetic force density in the radial direction at different current frequencies. It can be observed that when the frequency is small, the electromagnetic force density has an approximately linear relationship with the radius, which is consistent with the analytical formula [6]. However, when the frequency is greater than $8 \mathrm{~Hz}$, the electromagnetic force density is no longer linear with the radius due to the 'skin effect'.

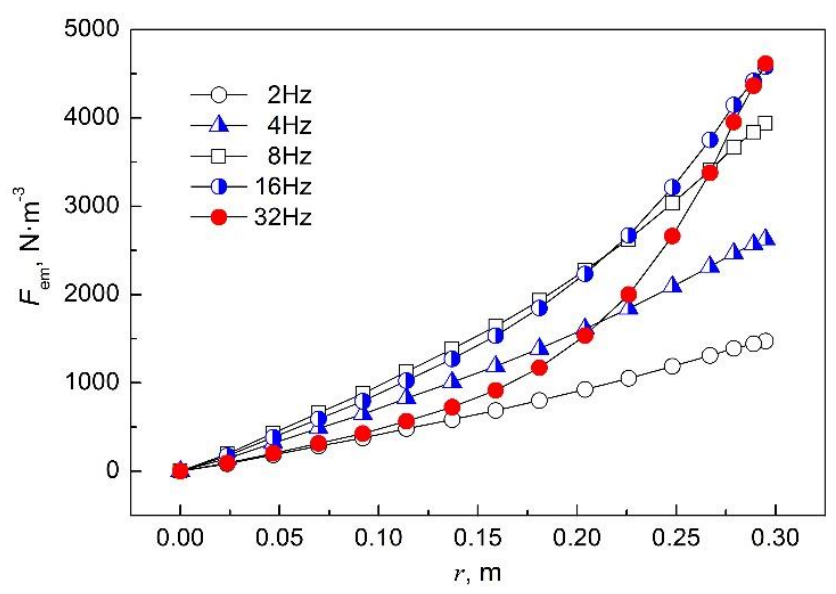

Figure 7. Electromagnetic force density profile as a function of radial distance at the middle plane of the stirrer for various current frequencies and $I=200 \mathrm{~A}$.

Figure 8 shows the effect of the current frequency on the magnetic flux density and induced current density. It is found that different variations appear at different positions of the $\Phi 600 \mathrm{~mm}$ round bloom. At the edge of the strand $(r=\mathrm{R})$, the frequency has little effect on the magnetic flux density, while the induced current density increases significantly with increasing frequency. At $r=\mathrm{R} / 2$ of the strand, an increase in frequency leads to a decrease in magnetic flux density, and the induced current density first increases and then decreases, and the maximum value appears at $14 \mathrm{~Hz}$. Since the electromagnetic force density is the cross-product of the magnetic flux density and the induced current 
density, it can be inferred from Figure 8 that there is a peak in the electromagnetic force density as a function of the current frequency.

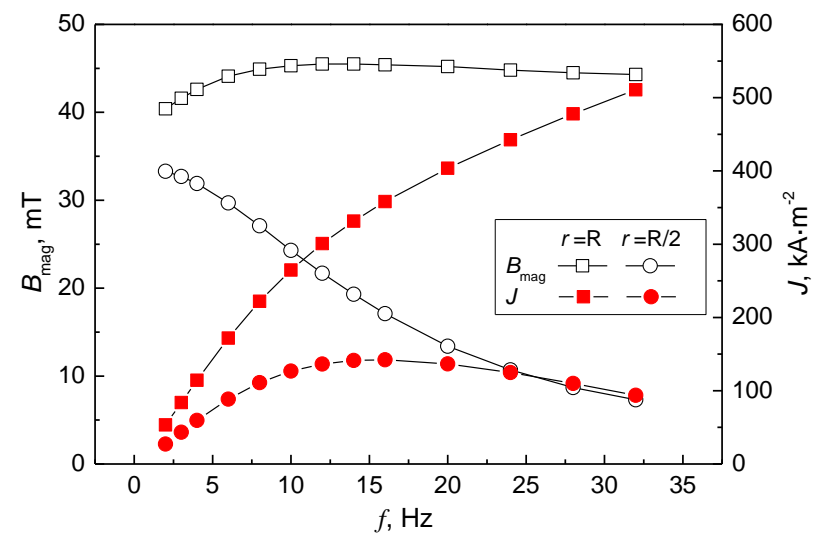

Figure 8. Effect of current frequency on the magnetic flux density and the induced current density at the middle plane of the stirrer for $I=200$ A.

Figure 9 reveals the relationship between the electromagnetic force density and the current frequency at different radii of the $\Phi 600 \mathrm{~mm}$ round bloom. At the same current intensity, the electromagnetic force density at the edge of the strand $(r=\mathrm{R})$ gradually increases with increasing frequency and remains basically constant until the frequency is greater than $16 \mathrm{~Hz}$. However, the electromagnetic force density at $r=\mathrm{R} / 2$ and $\mathrm{R} / 3$ of the strand reaches a maximum at a frequency of $10 \mathrm{~Hz}$ and then gradually decreases. Since the radius of the melt core is generally less than $\mathrm{R} / 2$ at the position where the strand is installed with F-EMS, the F-EMS of the $\Phi 600 \mathrm{~mm}$ round bloom has the highest stirring efficiency at a frequency of $10 \mathrm{~Hz}$.

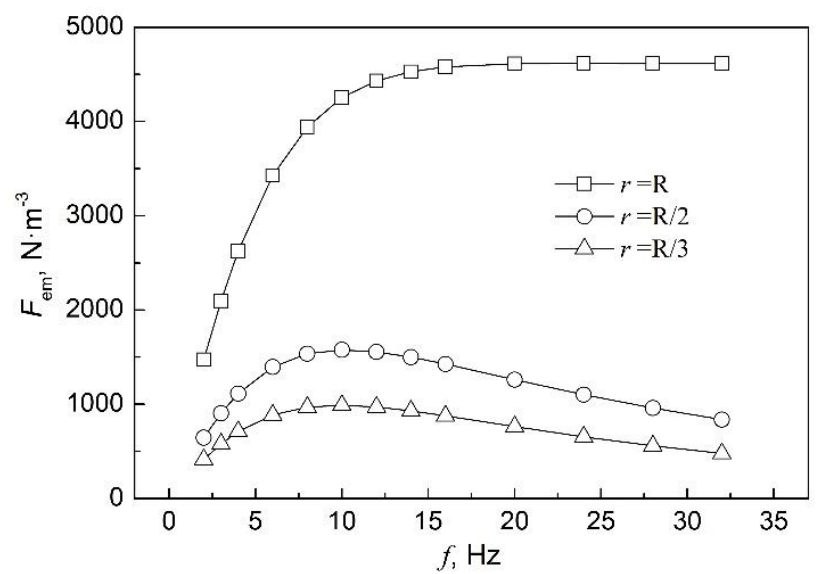

Figure 9. Effect of current frequency on the electromagnetic force density at different radii for $I=200 \mathrm{~A}$.

Figure 10 shows the effect of current intensity on the magnetic flux density and the electromagnetic force density. The former increases linearly with an increase in current intensity, while the latter has a quadratic relationship with the current intensity, which is consistent with the analytical formula deduced by Spitzer et al. [6]. 


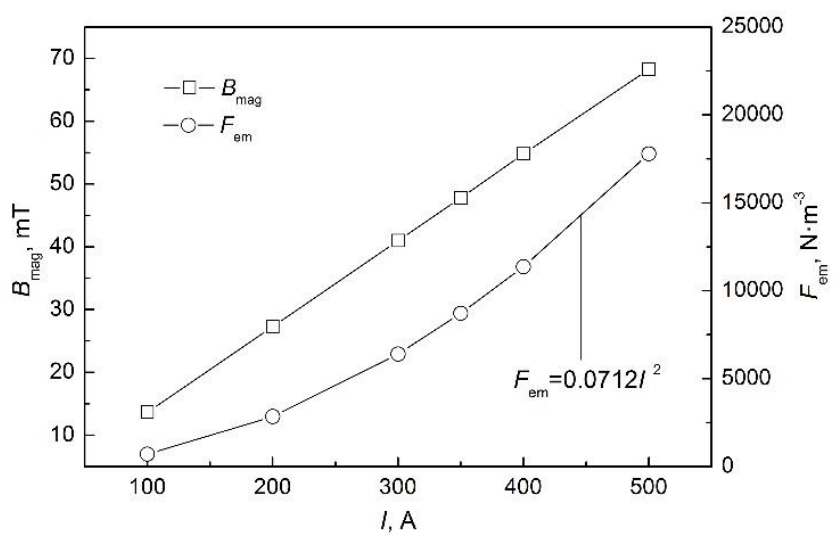

Figure 10. Effect of current intensity on the magnetic flux density and the electromagnetic force density at the middle plane of the stirrer for $f=4 \mathrm{~Hz}$.

\section{Conclusions}

The three-dimensional electromagnetic field in a continuous casting round bloom with F-EMS was studied numerically, and the influence of current intensity and frequency on the electromagnetic field was discussed. The following conclusions were obtained:

1. The Joule heat generated by F-EMS in a continuous casting strand is very small, and its influence on the secondary cooling heat transfer in the stirring zone can be ignored.

2. Since the electromagnetic force generated by F-EMS usually provides stirring action in a region less than $\mathrm{R} / 2$ of a round bloom, it is feasible to calculate it approximately using the analytical formula for the infinite-length stirring system.

3. With increase in current frequency, the electromagnetic force density at $R / 2$ and $R / 3$ of the strand first increases and then decreases, and it reaches a maximum at $10 \mathrm{~Hz}$. The results of the calculations show that the optimal current frequency of F-EMS is $10 \mathrm{~Hz}$ in continuous casting of a $\Phi 600 \mathrm{~mm}$ round bloom.

4. The magnetic flux density increases linearly with current intensity, while the electromagnetic force density has a quadratic relationship with current intensity.

Author Contributions: B.R., W.X., and Z.H. validated the model. B.R. performed the calculation. B.R., D.C., and H.W. analyzed and discussed the data. B.R. wrote the paper. D.C. and H.W. reviewed and edited the paper. B.R., W.X., and H.W. acquired the funding.

Funding: This research was funded by the National Natural Science Foundation of China, grant number 51674057; the Chongqing Research Program of Basic Research and Frontier Technology, grant numbers cstc2016jcyjA0142 and cstc2017jcyjAX0236; the Scientific and Technological Research Program of Chongqing Municipal Education Commission, grant numbers KJ1601326 and KJ1713343; and the Research Foundation of Chongqing University of Science \& Technology, grant number CK2016B19.

Conflicts of Interest: The authors declare no conflict of interest.

\section{References}

1. Barna, M.; Javurek, M.; Reiter, J.; Watzinger, J.; Kaufmann, B.; Kirschen, M. Continuous casting of round bloom strands with mould-electromagnetic stirring numerical simulations with a full coupling method. World Iron Steel 2012, 12, 29-33.

2. Hanley, P.J.; Kollberg, S.G. Electromagnetic methods for continuous casting. In The Making, Shaping and Treating of Steel, 11th ed.; Cramb, A.W., Ed.; Association for Iron \& Steel Technology: Pittsburgh, PA, USA, 2003; pp. 287-297, ISBN 978-0-930767-04-4.

3. Sun, H.; Li, L.; Cheng, X.; Qiu, W.; Liu, Z.; Zeng, L. Reduction in macrosegregation on $380 \mathrm{~mm} \times 490 \mathrm{~mm}$ bloom caster equipped combination M+F-EMS by optimising casting speed. Ironmak. Steelmak. 2015, 42, 439-449. [CrossRef] 
4. Mizukami, H.; Komatsu, M.; Kitagawa, T.; Kawakami, K. Effect of electromagnetic stirring at the final stage of solidification of continuously cast strand. Tetsu-to-Hagane 1984, 70, 194-200. [CrossRef]

5. Xiao, C.; Zhang, J.; Luo, Y.; Wei, X.; Wu, L.; Wang, S. Control of macrosegregation behavior by applying final electromagnetic stirring for continuously cast high carbon steel billet. J. Iron Steel Res. Int. 2013, 20, 13-20. [CrossRef]

6. Spitzer, K.H.; Dubke, M.; Schwerdtfeger, K. Rotational electromagnetic stirring in continuous casting of round strands. Metall. Trans. B 1986, 17, 119-131. [CrossRef]

7. Vynnycky, M. On an anomaly in the modeling of electromagnetic stirring in continuous casting. Metall. Mater. Trans. B 2018, 49, 399-410. [CrossRef]

8. Trindade, L.B.; Vilela, A.C.F.; Filho, Á.F.F.; Vilhena, M.T.M.B.; Soares, R.B. Numerical model of electromagnetic stirring for continuous casting billets. IEEE Trans. Magn. 2002, 38, 3658-3660. [CrossRef]

9. Yu, H.Q.; Zhu, M.Y. Influence of electromagnetic stirring on transport phenomena in round billet continuous casting mould and macrostructure of high carbon steel billet. Ironmak. Steelmak. 2012, 39, 574-584. [CrossRef]

10. Liu, H.; Xu, M.; Qiu, S.; Zhang, H. Numerical simulation of fluid flow in a round bloom mold with in-mold rotary electromagnetic stirring. Metall. Mater. Trans. B 2012, 43, 1657-1675. [CrossRef]

11. Jiang, D.; Zhu, M. Center segregation with final electromagnetic stirring in billet continuous casting process. Metall. Mater. Trans. B 2017, 48, 444-455. [CrossRef]

12. Marioni, L. Computational Modelling and Electromagnetic-CFD Coupling Incasting Processes; PSL Research University: Paris, France, 17 November 2017.

13. Davidson, P.A.; Hunt, J.C.R. Swirling recirculating flow in a liquid-metal column generated by a rotating magnetic field. J. Fluid Mech. 1987, 185, 67-106. [CrossRef]

14. Ren, B.Z.; Chen, D.F.; Wang, H.D.; Long, M.J.; Han, Z.W. Numerical simulation of fluid flow and solidification in bloom continuous casting mould with electromagnetic stirring. Ironmak. Steelmak. 2015, 42, 401-408. [CrossRef]

15. Barna, M.; Javurek, M.; Reiter, J.; Lechner, M. Numerical simulations of mould electromagnetic stirring for round bloom strands. Berg Huettenmaenn. Monatsh. 2009, 154, 518-522. [CrossRef]

16. Mao, B.; Ren, B.; Han, Z.; Cao, J.; Feng, K. Numerical simulation for heat transfer during solidification of round bloom continuous casting. Ind. Heat. 2012, 41, 50-53. [CrossRef]

17. Guo, G.; Ma, H.; Zhang, J.; Huang, L. The relationship of thermophysical properties to microstructure of high-carbon steel. Phys. Test. Chem. Anal. 2006, 42, 167-170.

18. Long, M.; Dong, Z.; Sheng, J.; Chen, D.; Chen, C. Universal secondary cooling structure for round blooms continuous casting of steels in various diameters. Steel Res. Int. 2015, 86, 154-162. [CrossRef] 